\title{
ON SOME REGULAR AND SINGULAR PROBLEMS OF BIRKHOFF INTERPOLATION
}

\author{
N. JHUNJHUNWALA and J. PRASAD \\ Department of Mathematics \\ California State University \\ Los Angeles, CA 90032
}

(Received September 4, 1992)

\begin{abstract}
Here we investigate the pure $(0,1, \cdots, r-2, r)$-interpolation problem on the zeros of $\left(1-x^{2}\right) P_{n}^{(\alpha)}(x)=\left(1-x^{2}\right) P_{n}^{(\alpha, \alpha)}(x), \alpha>-1$, where $P_{n}^{(\alpha, \alpha)}(x)$ is the Jacobi polynomial of degree $n$ with $\beta=\alpha$.
\end{abstract}

KEY WORDS AND PHRASES. Zeros, interpolation, incidence matrix, regular, singular.

1991 AMS SUBJECT CLASSIFICATION CODE. 41 A25.

\section{INTRODUCTION.}

Let $k$ and $l$ be natural numbers and let $E=E_{\ell}^{k}=\left(\epsilon_{i j}\right)(i=1,2, \cdots, k ; j=0,1, \cdots, l-1)$ be a matrix with $k$ rows and $l(l \geq k)$ columns having $\epsilon_{i j}=0$ or 1 , which are such that $\sum_{i, j} \epsilon_{i j}=1$ and no row is entirely composed of zeros. Let

$$
x_{1}<x_{2}<\cdots<x_{k}
$$

be increasing reals and $e_{l}^{k}=\left\{(i, j): \epsilon_{\imath}=1\right\}$. The reals $x_{i}$ and the incidence matrix $E$ describe the interpolation problem

$$
P^{(\jmath)}\left(x_{\imath}\right)=y_{i}^{(\jmath)}, \text { for }(i, j) \in e_{\ell}^{k}
$$

where $y_{i}^{(j)}$ are prescribed and the problem is to find the polynomial $P(x)$ of degree $\leq l-1$, which satisfies the condition (1.2). If $y_{i}^{(\jmath)}=0$ for $(i, j) \in e_{\ell}^{k}$ then the problem (1.2) is the homogeneous interpolation problem. Let $X=\left\{x_{i}\right\}_{1}^{k}$ be the interpolation nodes. We' say that $(E, X)$ is regular if (1.2) has a unique solution for all choices of reals $y_{i}^{(j)}$, and singular otherwise. If $P^{(j)}\left(x_{\mathfrak{a}}\right)=0$ for $(i, j) \epsilon e_{l}^{k}$, then $P(x)$ is said to be annihilated by $(E, X)$.

Turán and his associate [4] considered $E=E_{2 n}^{n}$ with $x_{1}, x_{2}, \cdots, x_{n}$ as the zeros of $\pi_{n}(x)=\left(1-x^{2}\right) P_{n-1}^{\prime}(x)$, where $P_{n}(x)$ is the Legendre polynomial of degree $n$ with normalization $P_{n}(1)=1$. Turán proved that $(E, X)$ is regular if $n$ is even and singular if $n$ is odd. Later, Varma ([5], [6]); Anderson and Prasad [1]; and Prasad and Anderson [3] considered different incidence matrices. Recently, Bajpai and Saxena [2] proved the following:

THEOREM A. If $E$ is the matrix of order $(n+2) \times(m+1)(n+2), m \geq 2$, with rows $(\underbrace{11 \ldots 1}_{m} 010 \cdots 0)$ and $X$ is the set of zeros of $\left(1-x^{2}\right) P_{n}(x), P_{n}(x)$ being the Legendre polynomial of degree $n$, then:

(i) if $m$ is even, $(E, X)$ is singular, and 
(ii) if $m$ is odd, $(E, X)$ is regular if $n$ is even and singular if $n$ is odd.

Let $X$ be the set of the zeros $\left\{x_{k}\right\}_{0}^{n+1}$ of $\left(1-x^{2}\right) P_{n}^{(\alpha)}(x)=\left(1-x^{2}\right) P_{n}^{(\alpha, \alpha)}(x), \alpha>-1$, where $P_{n}^{(\alpha, \alpha)}(x)$ is the Jacobi polynomial of degree $n$ with $\beta=\alpha$, such that

$$
-1=x_{n+1}<x_{n}<\cdots<x_{1}<x_{0}=1 .
$$

Our aim here is to prove the following:

THEOREM 1. Let $X$ be the set of the zeros of $\left(1-x^{2}\right) P_{n}^{(\alpha)}(x), \alpha>-1$, and $E$ be the incidence matrix given by

$$
E=E_{(n+2) \times(m+1)(n+2)}^{n+2}=\left(\begin{array}{ccccccc}
(1)_{m} & 0 & 1 & 0 & . & . & 0 \\
(1)_{m} & 0 & 1 & 0 & . & . & 0 \\
\cdot & . & . & . & . & . & . \\
. & . & . & . & . & . & . \\
(1)_{m} & 0 & 1 & 0 & . & . & 0
\end{array}\right)
$$

where $(1)_{m}$ means $m$ entries of 1 in that row. Let $m$ be an odd positive integer $\geq 3$, and $-1<\alpha<1$, then:

(i) if $n$ is odd then $(E, X)$ is singular.

(ii) if $n$ is even, $\alpha \neq \frac{m-2}{m+2}$, and $\alpha$ is such that $m-1-\alpha(m+2)$ is an odd positive integer then $(E, X)$ is singular and for all other values of $m-1-\alpha(m+2),(E, X)$ is regular.

(iii) if $n$ is even and $\alpha=\frac{m-2}{m+2}$, then $(E, X)$ is singular.

THEOREM 2. Let $X$ be the set of the zeros of $\left(1-x^{2}\right) P_{n}^{(\alpha)}(x), \alpha>-1$, and $E$ be the incidence matrix given by (1.1). Let $m$ be an even positive integer $\geq 2$, and $-1<\alpha<1$, then:

(i) If $n$ is odd then $(E, X)$ is singular.

(ii) If $n$ is even and $\alpha=\frac{m-2}{m+2},(0 \leq \alpha<1)$, then $(E, X)$ is singular.

(iii) If $n$ is even and $\alpha \neq \frac{m-2}{m+2}$, then $(E, X)$ is singular if $m-1-\alpha(m+2)$ is an odd positive integer and regular otherwise.

\section{SOME LEMMAS.}

Here we state and prove a few lemmas.

LEMMA 1. If $w_{n}(x)=P_{n}^{(\alpha)}(x), \alpha>-1, \lambda_{r}(x)=\left[\left(1-x^{2}\right) w_{n}^{2}(x)\right]^{r}, r=1,2, \cdots$ and $\left\{x_{k}\right\}_{1}^{n}$ are the zeros of $w_{n}(x)$ then:

The proof is obvious.

$$
\begin{aligned}
& {\left[w_{n}^{2 r}(x)_{x=x_{k}}^{(2 r)}=(2 r) !\left[w_{n}^{\prime}\left(x_{k}\right)\right]^{2 r}\right.} \\
& {\left[w_{n}^{2 r}(x)\right]_{i=x_{k}}^{(2 r+1)}=2 r(2 r+1) !(\alpha+1) x_{k}\left(1-x_{k}^{2}\right)^{-1}\left[w_{n}^{\prime}\left(x_{k}\right)\right]^{2 r}} \\
& =2 r(2 r+1) x_{k}(\alpha+1)\left(1-x_{k}^{2}\right)^{-1}\left[w_{n}^{2 r}(x)\right]_{x=x_{k}}^{(2 r)} \\
& \lambda_{r}^{(i)}\left(x_{k}\right)=\left\{\begin{array}{l}
0, i=0,1, \cdots, 2 r-1 \\
\left(1-x_{k}^{2}\right)^{r}(2 r) !\left[w_{n}^{\prime}\left(x_{k}\right)\right]^{2 r}, i=2 r,
\end{array}\right. \\
& \lambda_{r}^{(2 r+1)}\left(x_{k}\right)=2 r(2 r+1) ! \alpha x_{k}\left(1-x_{k}^{2}\right)^{r-1}\left[w_{n}^{\prime}\left(x_{k}\right)\right]^{2 r} \\
& =2 r(2 r+1) \alpha x_{k}\left(1-x_{k}^{2}\right)^{-1} \lambda_{r}^{(2 r)}\left(x_{k}\right) \text {. }
\end{aligned}
$$

LEMMA 2. Let $\delta_{2 r}(x)=\left(1-x^{2}\right)^{2 r}=\left(x^{2}-1\right)^{2 r}, r=1,2, \cdots$

Then:

$$
\delta_{2 r}^{(j)}( \pm 1)=\left\{\begin{array}{l}
0, i=0,1, \cdots, 2 r-1 \\
(2 r) ! 2^{2 r}, i=2 r
\end{array}\right.
$$




$$
\begin{aligned}
\delta_{2 r}^{(2 r+1)}(1)=2^{2 r}(2 r+1) ! r & =-\delta_{2 r}^{(2 r+1)}(-1) \\
& \delta_{2 r}^{2 r+1)}(1)=r(2 r+1) \delta_{2 r}^{(2 r)}(1)
\end{aligned}
$$

and

$$
\delta_{2 r}^{(2 r+1)}(-1)=-r(2 r+1) \delta_{2 r}^{(2 r)}(-1)
$$

The proof is obvious.

LEMMA 3. Let $F_{n}(x)=\left[\left(1-x^{2}\right) w_{n}(x)\right]^{m} q_{n+1}(x)$ be a polynomial of degree $\leq(n+2)(m+1)-1$, where $q_{n+1}(x)$ is a polynomial of degree $\leq n+1$, and let

$$
F_{n}^{(m+1)}\left(x_{k}\right)=0, k=0,1,2, \cdots, n+1 .
$$

Then, $q_{n+1}(x)$ satisfies the following conditions:

$$
\begin{gathered}
\left(1-x_{k}^{2}\right) q_{n+1}^{\prime}\left(x_{k}\right)+m(\alpha-1) x_{k} q_{n+1}\left(x_{k}\right)=0, k=1,2, \cdots, n ; \alpha>-1, \\
2 q_{n+1}^{\prime}(1)+m\left[\frac{n(n+2 \alpha+1)}{1+\alpha}+1\right] q_{n+1}(1)=0 \\
2 q_{n+1}^{\prime}(-1)-m\left[\frac{n(n+2 \alpha+1)}{1+\alpha}+1\right] q_{n+1}(-1)=0 .
\end{gathered}
$$

PROOF. Let $m=2 r$. Then

$$
F_{n}(x)=\lambda_{r}(x)\left[\left(1-x^{2}\right)^{r} q_{n+1}(x)\right]
$$

On using Leibnitz's formula and Lemma 1 one can easily see that for $k=1,2, \cdots, n$,

$$
F_{n}^{(2 r+1)}\left(x_{k}\right)=(2 r+1) \lambda_{r}^{(2 r)}\left(x_{k}\right)\left(1-x_{k}^{2}\right)^{r-1}\left[\left(1-x_{k}^{2}\right) q_{n+1}^{\prime}\left(x_{k}\right)+2 r x_{k}(\alpha-1) q_{n+1}\left(x_{k}\right)\right]
$$

To evaluate $F_{n}^{(2 r+1)}( \pm 1)$ we proceed as follows:

$$
F_{n}(x)=\delta_{2 r}(x)\left\{\left[w_{n}(x)\right]^{2 r} q_{n+1}(x)\right\} .
$$

Now, making use of Leibnitz formula and Lemma 2, we get

We know that

$$
\begin{aligned}
F_{n}^{(2 r+1)}(1) & =\delta_{2 r}^{(2 r+1)}(1)\left[w_{n}(1)\right]^{2 r} q_{n+1}(1)+ \\
& +\left(\begin{array}{c}
2 r+1 \\
2 r
\end{array}\right) \delta_{2 r}^{(2 r)}(1)\left\{2 r\left[w_{n}(1)\right]^{2 r-1} w_{n}^{\prime}(1) q_{n+1}(1)+\left[w_{n}(1)\right]^{2 r} q_{n+1}^{\prime}(1)\right\} .
\end{aligned}
$$

$$
\left(1-x^{2}\right) w_{n}^{\prime \prime}(x)-2(\alpha+1) x w_{n}^{\prime}(x)+n(n+2 \alpha+1) w_{n}(x)=0
$$

hence

$$
w_{n}^{\prime}(1)=\frac{n(n+2 \alpha+1)}{2(\alpha+1)} w_{n}(1), w_{n}(1)=\left(\begin{array}{c}
n+\alpha \\
n
\end{array}\right)
$$

So, from (2.13) and (2.15) it follows that

We also know that

$$
F_{n}^{(2 r+1)}(1)=2^{2 r-1}(2 r+1) !\left[w_{n}(1)\right]^{2 r}\left\{2 q_{n+1}^{\prime}(1)+2 r\left[1+\frac{n(n+2 \alpha+1)}{1+\alpha}\right] q_{n+1}(1)\right\} .
$$

$$
w_{n}(-1)=(-1)^{n} w_{n}(1),
$$




$$
w_{n}^{\prime}(-x)=(-1)^{n+1} w_{n}^{\prime}(x) .
$$

Further, using Leibnitz formula, Lemma $2,(2.17)$ and (2.18) one can easily verify that

$$
F_{n}^{(2 r+1)}(-1)=2^{2 r-1}(2 r+1) !\left[w_{n}(1)\right]^{2 r}\left\{2 q_{n+1}^{\prime}(-1)-2 r\left[1+\frac{n(n+2 \alpha+1)}{1+\alpha}\right] a_{n+1}(-1)\right\} .
$$

Next, let $m=2 r+1$. We now write

$$
F_{n}(x)=\lambda_{r}(x)\left[\left(1-x^{2}\right)^{r+1} w_{n}(x) q_{n+1}(x)\right] .
$$

Again, on using Leibnitz formula, Lemma 1 and (2.14), it follows that for $k=1,2, \cdots, n$,

$$
\begin{aligned}
F_{n}^{(2 r+2)}\left(x_{k}\right)=(2 r+1)(2 r+2)\left(1-x_{k}^{2}\right)^{r} w_{n}^{\prime}\left(x_{k}\right) & \lambda_{r}^{(2 r)}\left(x_{k}\right)\left[\left(1-x_{k}^{2}\right) q_{n+1}^{\prime}\left(x_{k}\right)\right. \\
& \left.+(2 r+1)(\alpha-1) x_{k} q_{n+1}\left(x_{k}\right)\right]
\end{aligned}
$$

Further, to compute $F_{n}^{(2 r+2)}( \pm 1)$, we write

$$
F_{n}(x)=\delta_{2 r}(x)\left[\left(1-x^{2}\right) w_{n}^{2 r+1}(x) q_{n+1}(x)\right]
$$

and use Leibnitz formula to get

$$
F_{n}^{(2 r+1)}(x)=\sum_{i=0}^{2 r+2}\left(\begin{array}{c}
2 r+2 \\
i
\end{array}\right) \delta_{2 r}^{(1)}(x)\left[\left(1-x^{2}\right) w_{n}^{2 r+1}(x) q_{n+1}(x)\right]^{(2 r+1-\imath)}
$$

On simplification using Lemma $2,(2.21)$ yields

$$
\begin{gathered}
F_{n}^{(2 r+2)}(1)=-(2 r+2) ! 2^{2 r} w_{n}^{2 r+1}(1)\left[2 q_{n+1}^{\prime}(1)+(2 r+1)\left\{\frac{n(n+2 \alpha+1)}{\alpha+1}+1\right\} q_{n+1}(1)\right] \\
F_{n}^{(2 r+2)}(-1)=(-1)^{n}(2 r+2) ! 2^{2 r} w_{n}^{2 r+1}(-1)\left[2 q_{n+1}^{\prime}(-1)-(2 r+1)\left\{\frac{n(n+2 \alpha+1)}{\alpha+1}+1\right\} q_{n+1}(-1)\right] .
\end{gathered}
$$

Hence the conditions

$$
F^{(m+1)}\left(x_{k}\right)=0, k=0,1,2, \cdots, n+1
$$

along with (2.12), (2.16), (2.19), (2.20), (2.22) and (2.23) imply (2.9), (2.10) and (2.11) for $m$ even or odd. This completes the proof of Lemma 3.

LEMMA 4. Let $q_{n+1}(x)$ be a polynomial of degree $\leq n+1$ which satisfies the following $n+2$ conditions:

$$
\begin{gathered}
\left(1-x_{k}^{2}\right) q_{n+1}^{\prime}\left(x_{k}\right)+m(\alpha-1) x_{k} q_{n+1}\left(x_{k}\right)=0, k=1,2, \cdots, n ; \dot{\alpha}>-1, \\
2 q_{n+1}^{\prime}(1)+m\left[\frac{n(n+2 \alpha+1)}{1+\alpha}+1\right] q_{n+1}(1)=0, \\
2 q_{n+1}^{\prime}(-1)-m\left[\frac{n(n+2 \alpha+1)}{1+\alpha}+1\right] q_{n+1}(-1)=0 .
\end{gathered}
$$

Then $q_{n+1}(x)$ satisfies the following equation:

$$
\left(1-x^{2}\right) q_{n+1}^{\prime}(x)+m(\alpha-1) x q_{n+1}(x)=c\left[x^{2}-\Delta(\alpha)\right] w_{n}(x),
$$

where $c$ is an arbitrary constant and

$$
\Delta(\alpha)=\frac{\frac{n(n+2 \alpha+1)+\alpha+1}{1+\alpha}\left[\left(\begin{array}{c}
n+\alpha \\
n
\end{array}\right)\left\{\frac{m(1-\alpha)+2}{1-\alpha}\right\}+1\right]+\left[2\left(\begin{array}{c}
n+\alpha \\
n
\end{array}\right)-1\right]}{\frac{n(n+2 \alpha+1)+\alpha+1}{1+\alpha}\left[\left(\begin{array}{c}
n+\alpha \\
n
\end{array}\right)\left\{\frac{m(1-\alpha)+2}{1-\alpha}\right\}+1\right]-\left[2\left(\begin{array}{c}
n+\alpha \\
n
\end{array}\right)+1\right]}, \alpha \neq 1
$$


PROOF. Due to (2.24), it follows that

$$
\left(1-x^{2}\right) q_{n+1}^{\prime}(x)+m(\alpha-1) x q_{n+1}(x)=\left(c x^{2}+d x+e\right) w_{n}(x),
$$

where $c, d$ and $e$ are constants. From (2.29), (2.15) and (2.17) we see that

$$
\begin{gathered}
m(\alpha-1) q_{n+1}(1)=(c+d+e)\left(\begin{array}{c}
n+\alpha \\
n
\end{array}\right), \\
-m(\alpha-1) q_{n+1}(-1)=(c-d+e)(-1)^{n}\left(\begin{array}{c}
n+\alpha \\
n
\end{array}\right) .
\end{gathered}
$$

Also, on differentiating (2.29) we have

$$
\begin{gathered}
\left(1-x^{2}\right) q_{n+1}^{\prime \prime}(x)+[m(\alpha-1)-2] x q_{n+1}^{\prime}(x)+m(\alpha-1) q_{n+1}(x)= \\
\left(c x^{2}+d x+e\right) w_{n}^{\prime}(x)+(2 c x+d) w_{n}(x) .
\end{gathered}
$$

Hence, from (2.32) we conclude that

$$
\begin{array}{r}
{[m(\alpha-1)-2] q_{n+1}^{\prime}(1)+m(\alpha-1) q_{n+1}(1)=(c+d+e) w_{n}^{\prime}(1)+(2 c+d) w_{n}(1),} \\
-[m(\alpha-1)-2] q_{n+1}^{\prime}(-1)+m(\alpha-1) q_{n+1}(-1)=(c-d+e) w_{n}^{\prime}(-1)+(-2 c+d) w_{n}(-1) .
\end{array}
$$

Further, from (2.30), (2.31), (2.15), (2.17), (2.18), (2.33) and (2.34) it follows that

$$
\begin{gathered}
{[m(\alpha-1)-2] q_{n+1}^{\prime}(1)=(c-e)\left(\begin{array}{c}
n+\alpha \\
n
\end{array}\right)+(c+d+e) \frac{n(n+2 \alpha+1)}{2(\alpha+1)}} \\
{[m(\alpha-1)-2] q_{n+1}^{\prime}(-1)=(-1)^{n}\left[(c-e)\left(\begin{array}{c}
n+\alpha \\
n
\end{array}\right)+(c-d+e) \frac{n(n+2 \alpha+1)}{2(\alpha+1)}\right]}
\end{gathered}
$$

Consequently, on substituting the values of $q_{n+1}(1), q_{n+1}^{\prime}(1), q_{n+1}(-1)$ and $q_{n+1}^{\prime}(-1)$ from the above equations into (2.25) and (2.26) and simplifying we get

$$
\begin{aligned}
& {\left[\left\{2\left(\begin{array}{c}
n+\alpha \\
n
\end{array}\right)-1\right\} c-d-\left\{2\left(\begin{array}{c}
n+\alpha \\
n
\end{array}\right)+1\right\} e\right]} \\
& +(c+d+e)\left[\frac{n(n+2 \alpha+1)}{1+\alpha}+1\right]\left[\left(\begin{array}{c}
n+\alpha \\
n
\end{array}\right)\left\{m+\frac{2}{1-\alpha}\right\}+1\right]=0 \\
& {\left[\left\{2\left(\begin{array}{c}
n+\alpha \\
n
\end{array}\right)-1\right\} c+d-\left\{2\left(\begin{array}{c}
n+\alpha \\
n
\end{array}\right)+1\right\} e\right]} \\
& +(c-d+e)\left[\frac{n(n+2 \alpha-1)}{1+\alpha}+1\right]\left[\left(\begin{array}{c}
n+\alpha \\
n
\end{array}\right)\left\{m+\frac{2}{1-\alpha}\right\}+1\right]=0 .
\end{aligned}
$$

Now, from (2.37) and (2.38) we see that $d=0$ and

$$
\begin{aligned}
& {\left[2\left(\frac{n+\alpha}{n}\right)-1\right] c-\left[2\left(\begin{array}{c}
n+\alpha \\
n
\end{array}\right)+1\right]} \\
& +(c+e)\left[\frac{n(n+2 \alpha+1)+1+\alpha}{1+\alpha}\right]\left[\left(\begin{array}{c}
n+\alpha \\
n
\end{array}\right)\left\{\frac{m(1-\alpha)+2}{1-\alpha}\right\}+1\right]=0
\end{aligned}
$$

which, on simplification, yields

$$
e=-\frac{\frac{n(n+2 \alpha+1)+\alpha+1}{1+\alpha}\left[\left(\begin{array}{c}
n+\alpha \\
n
\end{array}\right)\left\{\frac{m(1-\alpha)+2}{1-\alpha}\right\}+1\right]+\left[2\left(\begin{array}{c}
n+\alpha \\
n
\end{array}\right)-1\right]}{\frac{n(n+2 \alpha+1)+\alpha+1}{1+\alpha}\left[\left(\begin{array}{c}
n+\alpha \\
n
\end{array}\right)\left\{\frac{m(1-\alpha)+2}{1-\alpha}\right\}+1\right]-\left[2\left(\begin{array}{c}
n+\alpha \\
n
\end{array}\right)+1\right]} c
$$


or, using (2.28) we have

$$
e=-\Delta(\alpha) c
$$

This completes the proof of Lemma 4.

\section{PROOF OF THEOREM 1 AND THEOREM 2.}

Let $E$ be the incidence matrix given by (1.3) and let $X$ be the set of zeros of $\left(1-x^{2}\right) P_{n}^{(\alpha)}(x)=\left(1-x^{2}\right) w_{n}(x)$. Let $F_{n}(x)$ be a polynomial of degree $\leq(n+2)(m+1)-1$ annihilated by $(E, X)$. We have to ascertain if $F_{n}(x)$ is identically zero. Since

$$
\begin{gathered}
F_{n}\left(x_{k}\right)=F_{n}^{\prime}\left(x_{k}\right)=F_{n}^{\prime \prime}\left(x_{k}\right)=\cdots=F_{n}^{(m-1)}\left(x_{k}\right)=0, k=0,1, \cdots, n+1, \\
F_{n}(x)=\left[\left(1-x^{2}\right) w_{n}(x)\right]^{m} q_{n+1}(x),
\end{gathered}
$$

where $q_{n+1}(x)$ is a polynomial of degree $\leq n+1$. Further, since we have required that

$$
F_{n}^{(m+1)}\left(x_{k}\right)=0, k=0,1, \cdots, n+1,
$$

on account of Lemma $4, q_{n+1}(x)$ satisfies the following equation:

$$
\left(1-x^{2}\right) q_{n+1}^{\prime}(x)+m(\alpha-1) x q_{n+1}=c\left[x^{2}-\Delta(\alpha)\right] w_{n}(x),
$$

where $c$ is a numerical constant. Let

Further, it is well-known that

$$
q_{n+1}(x)=\sum_{k=0}^{n+1} a_{k} w_{k}(x) .
$$

$$
\left(1-x^{2}\right) w_{n}^{\prime}(x)=-n x w_{n}(x)+(n+\alpha) w_{n-1}(x) .
$$

Now, from (3.1), (3.2) and (3.3), on simple computations, it follows that

$$
\begin{gathered}
\sum_{k=1}^{n+2} a_{k-1}[m(\alpha-1)-k+1] \frac{k(k+2 \alpha)}{(k+\alpha)(2 k+2 \alpha-1)} w_{k}(x) \\
+\sum_{k=0}^{n} a_{k+1}(k+\alpha+1)\left[1+\frac{m(\alpha-1)-k-1}{2 k+2 \alpha+3}\right] w_{k}(x)=c\left[x^{2}-\Delta(\alpha)\right] w_{n}(x) .
\end{gathered}
$$

Also, we know that

$$
x w_{n}(x)=\frac{(n+1)(n+2 \alpha+1)}{(2 n+2 \alpha+1)(n+\alpha+1)} w_{n+1}(x)+\frac{(n+\alpha)}{(2 n+2 \alpha+1)} w_{n-1}^{\prime}(x) .
$$

Repeated application of (3.5) in (3.4), on simplification, yields

$$
\begin{gathered}
\sum_{k=0}^{n} a_{k+1} \frac{(k+\alpha+1)[k+\alpha(m+2)+2-m]}{(2 k+2 \alpha+3)} w_{k}(x) \\
+\sum_{k=1}^{n+2} a_{k-1} \frac{[m(\alpha-1)-k+1] k(k+2 \alpha)}{(k+\alpha)(2 k+2 \alpha-1)} w_{k}(x) \\
=A w_{n-2}(x)+B w_{n}(x)+C w_{n+2}(x),
\end{gathered}
$$

where

$$
\begin{gathered}
A=\frac{(n+\alpha)(n+\alpha-1)}{(2 n+2 \alpha+1)(2 n+2 \alpha-1)} c \\
B=\left[\frac{(n+1)(n+2 \alpha+1)}{(2 n+2 \alpha+1)(2 n+2 \alpha+3)}+\frac{n(n+2 \alpha)}{(2 n+2 \alpha+1)(2 n+2 \alpha-1)}-\Delta(\alpha)\right] c
\end{gathered}
$$




$$
C=\frac{(n+1)(n+2 \alpha+1)(n+2)(n+2 \alpha+2)}{(n+\alpha+1)(n+\alpha+2)(2 n+2 \alpha+1)(2 n+2 \alpha+3)} c .
$$

Consequently, we obtain

$$
\begin{aligned}
& \frac{(1+\alpha)[\alpha(m+2)+2-m]}{2 \alpha+3} a_{1}=0 \\
& \frac{(k+1+\alpha)[k+\alpha(m+2)+2-m]}{(2 k+2 \alpha+3)} a_{k+1}+\frac{k(k+2 \alpha)[m(\alpha-1)-k+1]}{(k+\alpha)(2 k+2 \alpha-1)} a_{k-1}=0, \\
& k=1,2, \cdots, n-4, n-3, \\
& \frac{(n+\alpha-1)[n+\alpha(m+2)-m]}{(2 k+2 \alpha-1)} a_{n-1}+\frac{[m(\alpha-1)-n+3](n-2)(n+2 \alpha-2)}{(n+\alpha-2)(2 n+2 \alpha-5)} a_{n-3}=A, \\
& \frac{(n+\alpha)[n+\alpha(m+2)+1-m]}{(2 n+2 \alpha+1)} a_{n}+\frac{[m(\alpha-1)-n+2](n-1)(n+2 \alpha-1)}{(n+\alpha-1)(2 n+2 \alpha-3)} a_{n-2}=0, \\
& \frac{(n+\alpha+1)[n+\alpha(m+2)+2-m]}{(2 n+2 \alpha+3)} a_{n+1}+\frac{[m(\alpha-1)-n+1] n(n+2 \alpha)}{(n+\alpha)(2 n+2 \alpha-1)} a_{n-1}=B, \\
& \frac{(n+2 \alpha+1)(n+1)[m(\alpha-1)-n]}{(n+\alpha+1)(2 n+2 \alpha+1)} a_{n}=0,
\end{aligned}
$$

and

$$
\frac{(n+2)(n+2 \alpha+2)[m(\alpha-1)-n-1]}{(n+\alpha+2)(2 n+2 \alpha+3)} a_{n+1}=C
$$

Let $m$ be an odd positive integer $\geq 3$ :

(i) If $n$ is odd, $-1<\alpha<1$, and $\alpha$ is such that $m-1-\alpha(m+2)$ is an even positive integer then

$$
a_{n}=a_{n-2}=\cdots=a_{3}=a_{1}=0
$$

and

$$
a_{0}=a_{2}=\cdots=a_{m-3-\alpha(m+2)}=0
$$

but $a_{m-1-\alpha(m+2)}, a_{m+1-\alpha(m+2)}, \cdots, a_{n+1}$ are note necessarily zero. Hence, $q_{n+1}(x)$ is not identically zero. If $n$ is odd, $-1<\alpha<1$, and $\alpha$ is such that $m-1-\alpha(m+2)$ is an even negative integer then

$$
a_{n}=a_{n-2}=\cdots \cdots=a_{3}=a_{1}=0
$$

and $a_{0}, a_{2}, \cdots, a_{n-3}$ are not necessarily zero. Hence, $q_{n+1}(x)$ is not identically zero.

If $n$ is odd, $-1<\alpha<1$, and $\alpha$ is such that $m-1-\alpha(m+2)$ is an odd integer or a fraction then

$$
a_{n}=a_{n-2}=\cdots=a_{3}=a_{1}=0
$$

but $a_{0}, a_{2}, \cdots, a_{n-3}, a_{n-1}$ and $a_{n+1}$ are not all zero. Hence, $q_{n+1}(x)$ is not identically zero. So, it follows that $(E, X)$ is singular if $n$ is odd.

(ii) If $n$ is even, $-1<\alpha<1, \alpha \neq \frac{m-2}{m+2}$, and $\alpha$ is such that $m-1-\alpha(m+2)$ is an odd positive integer then

$$
a_{n}=a_{n-2}=\cdots=a_{2}=a_{0}=0
$$

and 


$$
a_{1}=a_{3}=\cdots=a_{m-3-\alpha(m+2)}=0,
$$

but $a_{m-1-\alpha(m+2)}, a_{m+1-\alpha(m+2)}, \cdots, a_{n+1}$ are not necessarily zero. Hence, $q_{n+1}(x)$ is not identically zero. If $n$ is even, $-1<\alpha<1, \alpha \neq \frac{m-2}{m+2}, \alpha$ is such that $m-1-\alpha(m+2)$ is an odd negative integer then

$$
a_{n}=a_{n-2}=\cdots=a_{2}=a_{0}=0 .
$$

Noting that $a_{1}=0$ we conclude that

$$
a_{1}=a_{3}=\cdots=a_{n-3}=0 .
$$

Recalling the equations for $a_{n-1}$ and $a_{n+1}$ and substituting the values of $A, B$, and $C$ it can be easily verified that $c=0$. So, $a_{n-1}$ and $a_{n+1}$ are also zero. Hence, $q_{n+1}(x)$ is identically zero. If $n$ is even, $-1<\alpha<1, \alpha \neq \frac{m-2}{m+2}$, and $\alpha$ is such that $m-1-\alpha(m+2)$ is an even positive integer then

$$
a_{n}=a_{n-2}=\cdots=a_{2}=a_{0}=0
$$

and

$$
a_{1}=a_{3}=\cdots=a_{n-3}=a_{n-1}=a_{n+1}=0 .
$$

Hence, $q_{n+1}(x)$ is identically zero.

If $n$ is even, $-1<\alpha<1, \alpha \neq \frac{m-2}{m+2}$, and $\alpha$ is such that $m-1-\alpha(m+2)$ is an even negative integer then

$$
a_{n}=a_{n-2}=\cdots=a_{2}=a_{0}=0
$$

and

$$
a_{1}=a_{3}=\cdots=a_{n-3}=a_{n-1}=a_{n+1}=0 .
$$

Hence, $q_{n+1}(x)$ is identically zero.

If $n$ is even, $-1<\alpha<1, \alpha \neq \frac{m-2}{m+2}$, and $\alpha$ is such that $m-1-\alpha(m+2)$ is a fraction then

$$
a_{n}=a_{n-2}=\cdots=a_{2}=a_{0}=0
$$

and

$$
a_{1}=a_{3}=\cdots=a_{n-3}=a_{n-1}=a_{n+1}=0 .
$$

Hence, $q_{n+1}(x)$ is identically zero. Consequently, if $n$ is even, $-1<\alpha<1, \alpha \neq \frac{m-2}{m+2}$, and $\alpha$ is such that $m-1-\alpha(m+2)$ is an odd positive integer then $(E, X)$ is singular and for all other values of $m-1-\alpha(m+2),(E, X)$ is regular.

(iii) If $n$ is even, $\alpha=\frac{m-2}{m+2}$, and $\alpha$ is such that $m-1-\alpha(m+2)$ is a negative integer then

$$
a_{n}=a_{n-2}=\cdots=a_{2}=a_{0}=0
$$

and $a_{1}, a_{3}, \cdots, a_{n-3}$ are not necessarily zero. Hence, $q_{n+1}(x)$ is not identically zero.

If $n$ is even, $\alpha=\frac{m-2}{m+2}$, and $\alpha$ is such that $m-1-\alpha(m+2)$ is an odd positive integer then

$$
a_{n}=a_{n-2}=\cdots=a_{2}=a_{0}=0
$$

and

$$
a_{1}=a_{3}=\cdots \cdots=a_{m-3-\alpha(m+2)}=0
$$


but $a_{m-1-\alpha(m+2)}, a_{m+1-\alpha(m+2)}, \cdots, a_{n-1}$ are not necessarily zero. Hence, $q_{n+1}(x)$ is not identically zero. If $n$ is even, $\alpha=\frac{m-2}{m+2}$, and $\alpha$ is such that $m-1-\alpha(m+2)$ is an even positive integer then

$$
a_{n}=a_{n-2}=\cdots=a_{2}=a_{0}=0
$$

and $a_{1}, a_{3}, \cdots, a_{n-3}$ are not necessarily zero. Hence, $q_{n+1}(x)$ is not identically zero.

Consequently, in this case, $(E, X)$ is singular.

This completes the proof of Theorem 1.

Next, let $m$ be an even positive integer $\geq 2$, and $-1<\alpha<1$ :

(i) If $n$ is odd then

$$
a_{n}=a_{n-2}=\cdots=a_{3}=a_{1}=0
$$

but not all $a_{0}, a_{2}, \cdots, a_{n+1}$ are zero. Hence, $q_{n+1}(x)$ is not identically zero. So, $(E, X)$ is singular.

(ii) If $n$ is even and $\alpha=\frac{m-2}{m+2}, 0 \leq \alpha<1$, then

$$
a_{n}=a_{n-2}=\cdots=a_{2}=a_{0}=0
$$

but $a_{1}, a_{3}, \cdots, a_{n-3}$ are not necessarily zero. Hence $q_{n+1}(x)$ is not identically zero. Consequently, $(E, X)$ is singular.

(iii) If $n$ is even, $\alpha \neq \frac{m-2}{m+2}$, and $\alpha$ is such that $m-1-\alpha(m+2)$ is an odd positive integer then

$$
a_{n}=a_{n-2}=\cdots=a_{2}=a_{0}=0
$$

and

$$
a_{1}=a_{3}=\cdots=a_{m-3}-\alpha(m+2)=0,
$$

but $a_{m-1-\alpha(m+2)}, a_{m+1-\alpha(m+2)}, \cdots, a_{n+1}$ are not necessarily zero. Hence $q_{n+1}(x)$ is not identically zero.

If $n$ is even, $\alpha \neq \frac{m-2}{m+2}$ and $\alpha$ is such that $m-1-\alpha(m+2)$ is an odd negative integer then

$$
a_{n}=a_{n-2}=\cdots=a_{2}=a_{0}=0
$$

and since $k+\alpha(m+2)+2-m$ is never zero for even values of $k$ hence

$$
a_{1}=a_{3}=\cdots=a_{n-3}=a_{n-1}=a_{n+1}=0 .
$$

So, $q_{n+1}(x)$ is identically zero.

If $n$ is even, $\alpha \neq \frac{m-2}{m+2}$ and $\alpha$ is such that $m-1-\alpha(m+2)$ is an even positive integer then

$$
a_{n}=a_{n-2}=\cdots=a_{2}=a_{0}=0
$$

and also $a_{1}=0$ so that $k+\alpha(m+2)+2-m$ is never zero for even values of $k$ hence

$$
a_{1}=a_{3}=\cdots=a_{n-3}=a_{n-1}=a_{n+1}=0 .
$$

Consequently, $q_{n+1}(x)$ is identically zero.

If $n$ is even, $\alpha \neq \frac{m-2}{m+2}$ and $\alpha$ is such that $m-1-\alpha(m+2)$ is an even negative integer then

$$
a_{n}=a_{n-2}=\cdots=a_{2}=a_{0}=0
$$

and also $a_{1}=0$ so that $k+\alpha(m+2)+2-m$ is never zero hence

$$
a_{1}=a_{3}=a_{5}=\cdots=a_{n-3}=a_{n-1}=a_{n+1}=0 .
$$


Consequently, $q_{n+1}(x)$ is identically zero.

If $n$ is even, $\alpha \neq \frac{m-2}{m+2}$ and $\alpha$ is such that $m-1-\alpha(m+2)$ is a fraction then

$a_{n}=a_{n-2}=\cdots=a_{0}=0$,

$a_{1}=0$, so $a_{1}=a_{3}=a_{5}=\cdots=a_{n-3}=a_{n-1}=a_{n+1}=0$.

Therefore, $q_{n+1}(x)$ is identically zero. Consequently, $(E, X)$ is singular if $m-1-\alpha(m+2)$ is an odd positive integer and regular otherwise. This completes the proof of Theorem 2.

In conclusion, it is worthwhile to mention that $\mathrm{H}$. Windauer [7] has also considered the modified $(0,1, \cdots, r-2, r)$-interpolation problem on the zeros of $\left(1-x^{2}\right) P_{n}^{(\alpha)}(x), \alpha>-1$, and $(0,1, \cdots, r-2, r)$-interpolation problem on the zeros of $P_{n}^{(\alpha)}(x), \alpha>-1$. As is evident, we have addressed the $(0,1, \cdots, r-2, r)$-interpolation problem on the zeros of $\left(1-x^{2}\right) P_{n}^{(\alpha)}(x), \alpha>-1$.

\section{REFERENCES}

1. ANDERSON, C.H. \& PRASAD, J., On the non-existence of some interpolatory polynomials, Internat. J. Math. \& Math. Sci. 9 (1986), 753-756.

2. BAJPAI, P. \& SAXENA, R.B., Some regular and singular pairs of Birkhoff interpolation, Demonstratio Mathematica XXII, No. 3, (1989), 583-597.

3. PRASAD, J. \& ANDERSON, C.H., Some remark on non-existence of interpolation polynomials, Mathematicae Notae - AÑO XXIX, (1981/82), 67-72.

4. SURÁNYI, J. \& TURÁN, P., Notes on interpolation I, Acta Math. Acad. Sci. Hung. 6, (1955), 66-79.

5. VARMA, A.K., Non-existence of interpolatory polynomials, Publ. Math. (Debrecan) 15, (1968), 75-77.

6. VARMA, A.K., An analogue of a problem of J. Balaźs and P. Turán III, Trans. Amer. Math. Soc. 146, (1969), 107-120.

7. WINDAUER, H., Existenz und Eindeutigkeit bei der Hermite-Bikhoff-Interpolation (Dissertation). Bochum, Ruhr-Universitaet, 1972. 


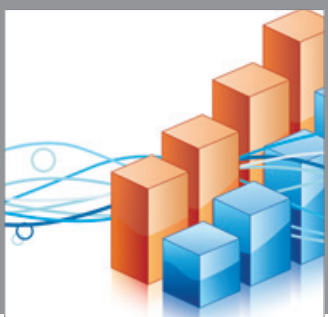

Advances in

Operations Research

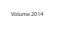

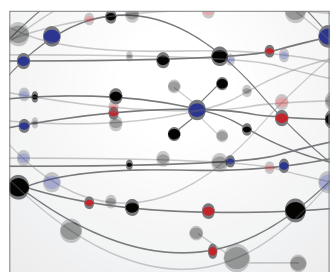

\section{The Scientific} World Journal
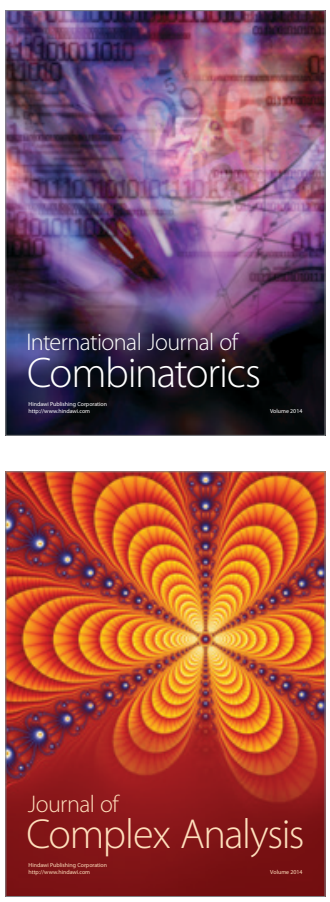

International Journal of

Mathematics and

Mathematical

Sciences
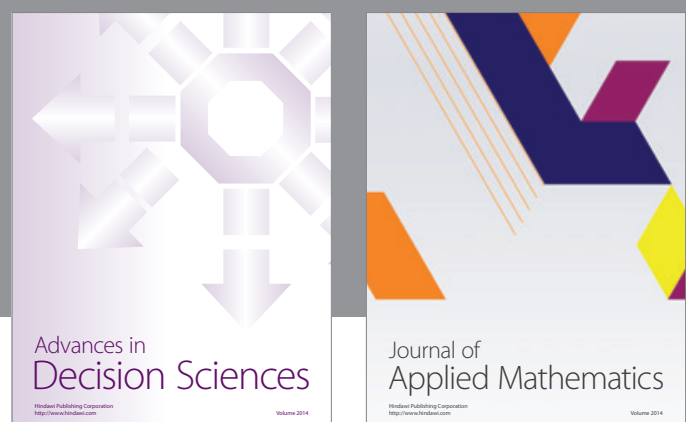

Journal of

Applied Mathematics
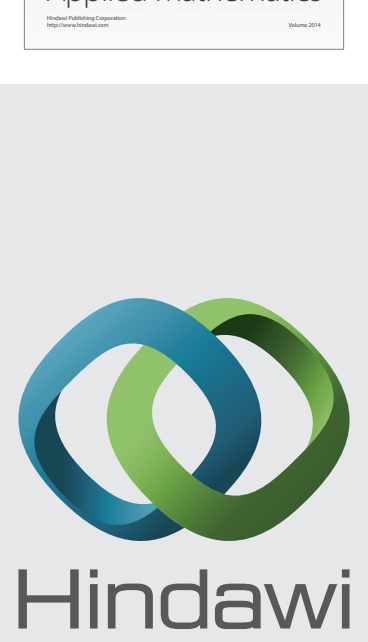

Submit your manuscripts at http://www.hindawi.com
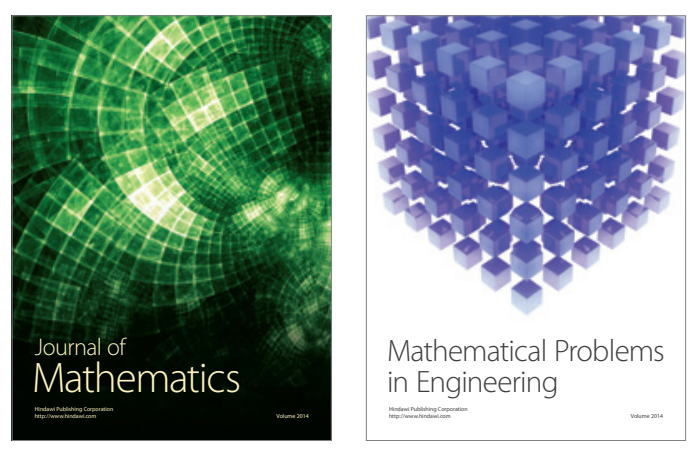

Mathematical Problems in Engineering
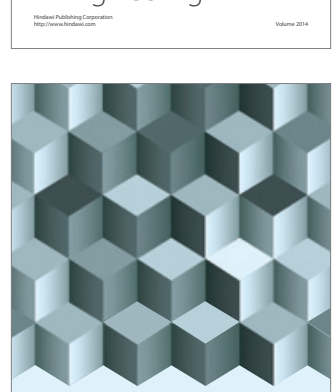

Journal of

Function Spaces
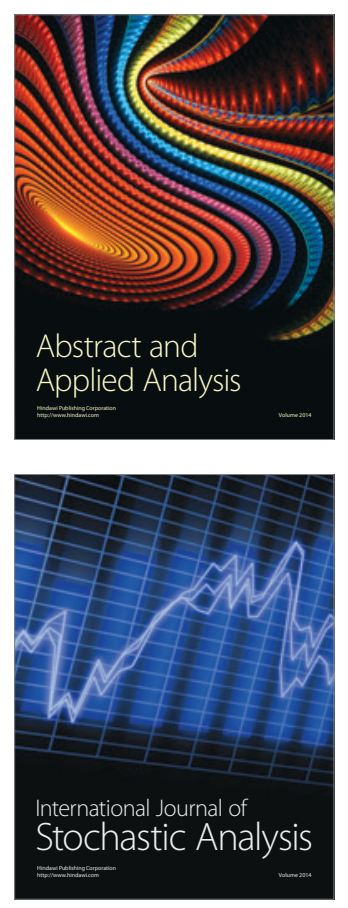

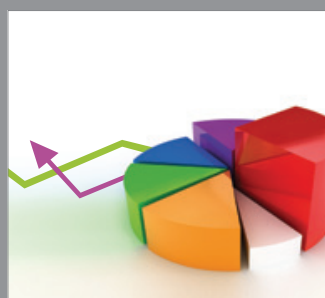

ournal of

Probability and Statistics

Promensencen
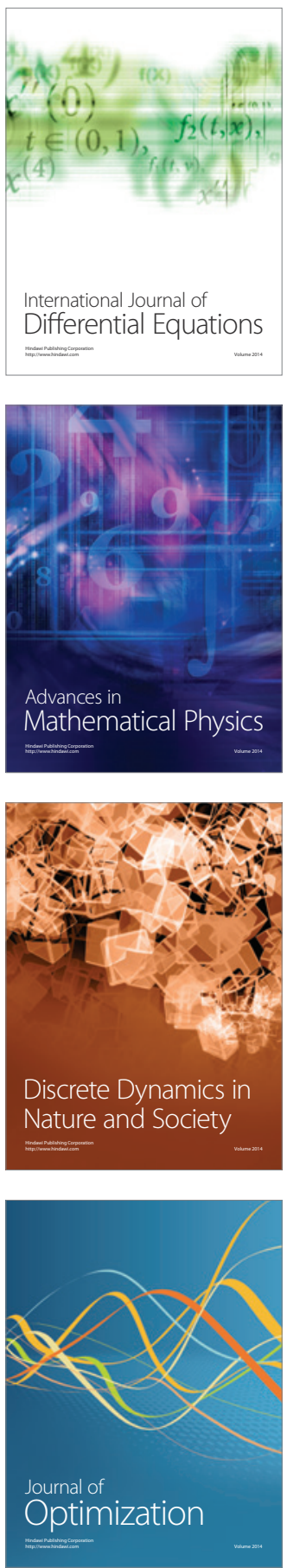